\title{
LVI. A note on van der Waals' equation
}

\section{Harold Hilton}

To cite this article: Harold Hilton (1901) LVI. A note on van der Waals' equation , Philosophical Magazine Series 6, 1:5, 579-589, DOI: 10.1080/14786440109462648

To link to this article: http://dx.doi.org/10.1080/14786440109462648

曲 Published online: 08 Jun 2010.

Submit your article to this journal 준

Џll Article views: 2

Q View related articles $₫$ 


\section{$\left[\begin{array}{ll}579\end{array}\right]$ \\ LVI. A Note on van der Waals' Equation. By Harold Hilton*.}

$\mathrm{B}^{\text {EARING in mind the great importance of van der Waals' }}$ B equation, it seems worth while to collect the various mathematical properties of the family of curves which is the graphical representation of the equation, together with some accurate tracings of several members of the family.

The form of van der Waals' equation we shall consider is

$$
y=\frac{8 \theta}{3 x-1}-\frac{3}{x^{2}}, \text {. . . . . . }
$$

or, as it may be written,

$$
3 y x^{3}-(y+8 \theta) x^{2}+9 x-3=0,
$$

where $x, y$, and $\theta$ are respectively the "reduced" volume, pressure, and temperature; $i$. e., the volume, pressure, and temperature expressed with the critical volume, pressure, and temperature respectively as units.

The equation ( $\alpha$ ) may be considered as representing a family of curves of the fourth degree, of which $\theta$ is the parameter.

The curve $(\alpha)$ cuts the axis of $x$ in finite points, where $8 \theta x^{2}-9 x+3=0$; the roots of this equation are unreal if $\theta>\frac{27}{32}$; both equal to ${ }_{3}^{2}$ if $\theta=\frac{27}{32}$; both positive and $>\frac{1}{3}$, if $\theta$ lies between $\frac{27}{32}$ and 0 ; one root is infinite if $\theta=0$; both roots are real, one lying between $-\infty$ and 0 , and the other between 0 and $\frac{1}{3}$, if $\theta$ is negative. The curve cuts the axis of $y$ in no finite point. The curve has the axis of $x$ as an ordinary asymptote, and a triple point at infinity in the direction of the axis of $y$, at which $x=\frac{1}{3}$ is the tangent to one branch, and the tangents to the other two branches coincide in $x=0$.

Hence to every value of $x$ there can be at most one finite value of $y$; and to every value of $y$ there can be at most three finite values of $x$ of which two may be imaginary.

If $\theta$ is positive, the curve has a branch between $x=\infty$ and $x=\frac{1}{3}$, touching $y=0$ and $x=\frac{1}{3}$ at infinity ; so that $y$ is in each case positive; a branch lying between $x=\frac{1}{3}$ and $x=0$, and touching these lines at infinity, so that $y$ is in each case negative (in fact for most positive values of $\theta$ this branch lies altogether so far on the negative side of $y=0$ that it cannot well be drawn on any diagram, and hence does not appear in fig. 1); and a branch on the negative side of $x=0$ touching

* Communicated by the Author. 
$x=0$ and $y=0$ at infinity, so that $x$ and $y$ for the branch are always negative.

If $\theta=0$, the curve breaks up into the straight line $x=\frac{1}{3}$ and the curve $y x^{2}+3=0$; these two branches meet at the point $\left(\frac{1}{3},-27\right)$.

If $\theta$ is negative, there is a branch between $x=\infty$ and $x=\frac{1}{3}$ touching $y=0$ and $x=\frac{1}{3}$ at infinity, $y$ for this branch being always negative; a branch between $x=\frac{1}{3}$ and $x=0$ touching these lines at infinity, so that near $x=\frac{1}{3}$ the ordinate of the curve is positive and near $x=0$ negative (the branch crosses the axis of $x$ and has an inflexion, but cannot in a diagram be well distinguished from a straight line); and a branch on the negative side of $x=0$ for which $y$ is negative near $x=0$ and positive near $x=-\infty$ (the branch crosses the axis of $x$, has a tangent parallel to this axis, and an inflexion).

Any member of the family can be readily traced by taking a series of values for $x$ and calculating the corresponding values of $y$. Fig. 1 shows as much of the curves $\theta=\frac{1}{2}$ and $\theta=-1$ as can be conveniently put on a diagram. Fig. 2 shows the part of the curves lying between $x=\frac{1}{3}$ and $x=5$ (which is the part interesting from a physical point of view) for 19 positive values of $\theta$.

The tables given below show the values of $y$ calculated to three places of decimals from which the figures were drawn.

Any member of the family is of degree 4, of class 5, and of deficiency zero; it has two double points and one cusp (coinciding in the multiple point at infinity) ; two bitangents; and four inflexions (of which two are always imaginary, and the other two are also imaginary if $\left.\theta>\frac{2187}{2048}\right)$.

The area included between any curve of the family and the lines $y=0, x_{1}=0, x_{2}=0$, is

The orthogonal trajectory of the system is

$$
\frac{8 \theta}{3} \log \frac{3 x_{2}-1}{3 x_{1}-1}+\frac{3}{x_{2}}-\frac{3}{x_{1}} \text {. }
$$

$$
x^{3}(3 x-1)=\frac{d y}{d x}\left(3 x^{3} y-9 x+6\right) .
$$

The radius of curvature at any point is

$$
\frac{\left\{x^{6}(3 x-1)^{2}+\left(24 \theta x^{3}-6 \overline{3 x-1}\right)^{2}\right\}^{\frac{3}{2}}}{18 x^{5}(3 x-1)^{2}\left[8 \theta x^{4}-(3 x-1)^{3}\right]}
$$

if the tangent at the point is parallel to $y=0$, this simplifies into

$$
\frac{x^{4}(3 x-1)}{18(1-x)}
$$


Mr. H. Hilton on van der W'aals' Equation. $\quad 581$

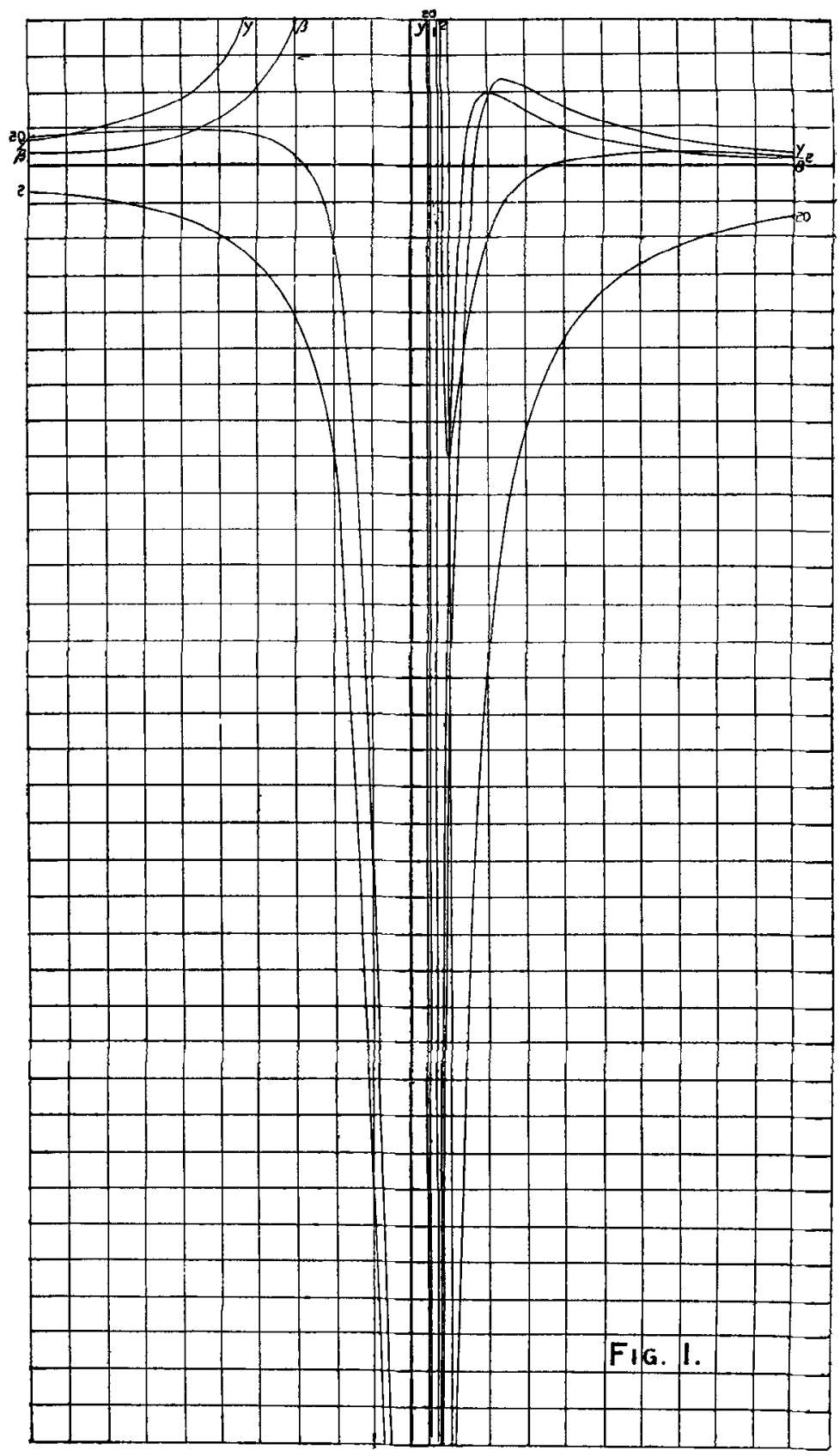


582 Mr. H. Hilton on van der Waals' Equation.

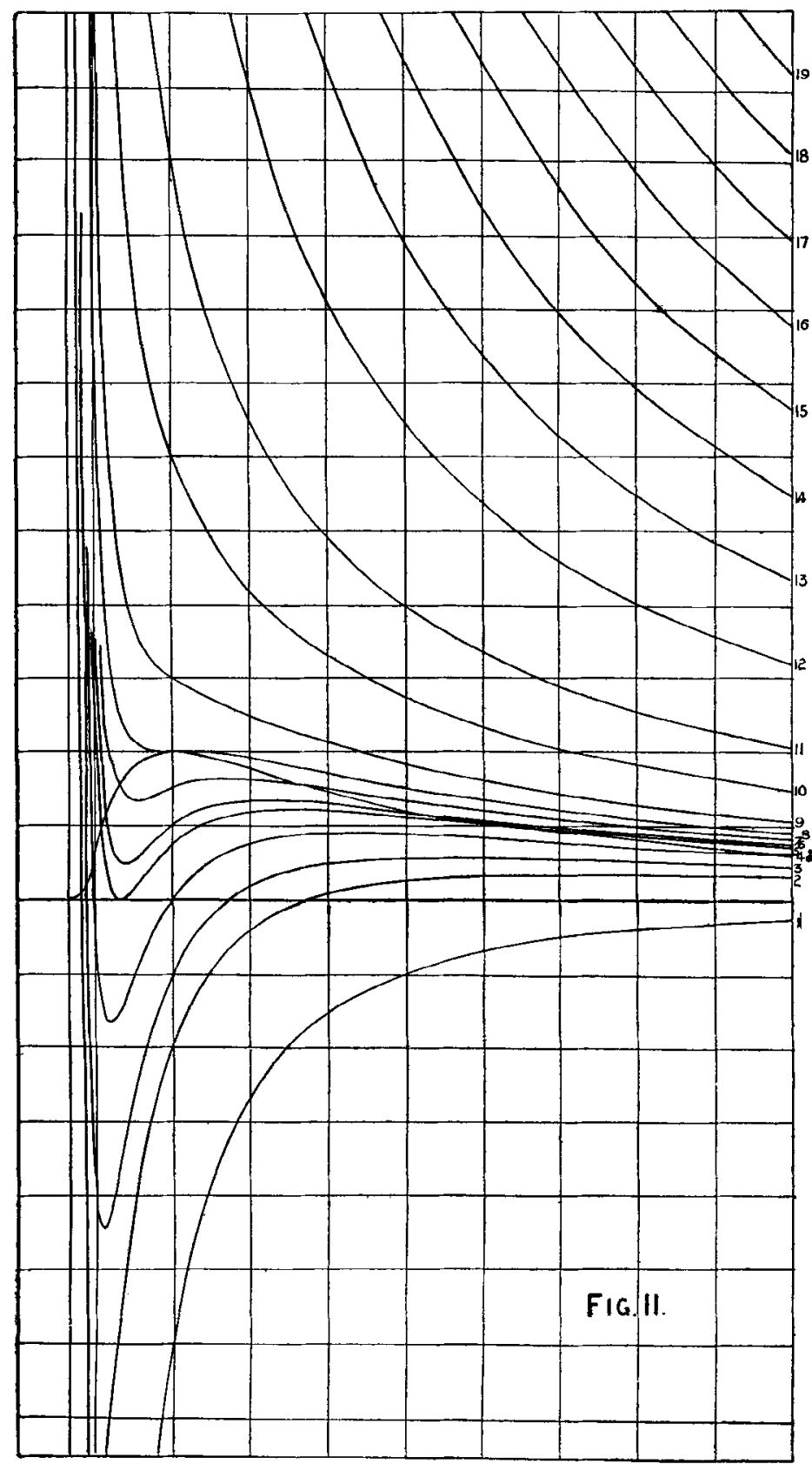




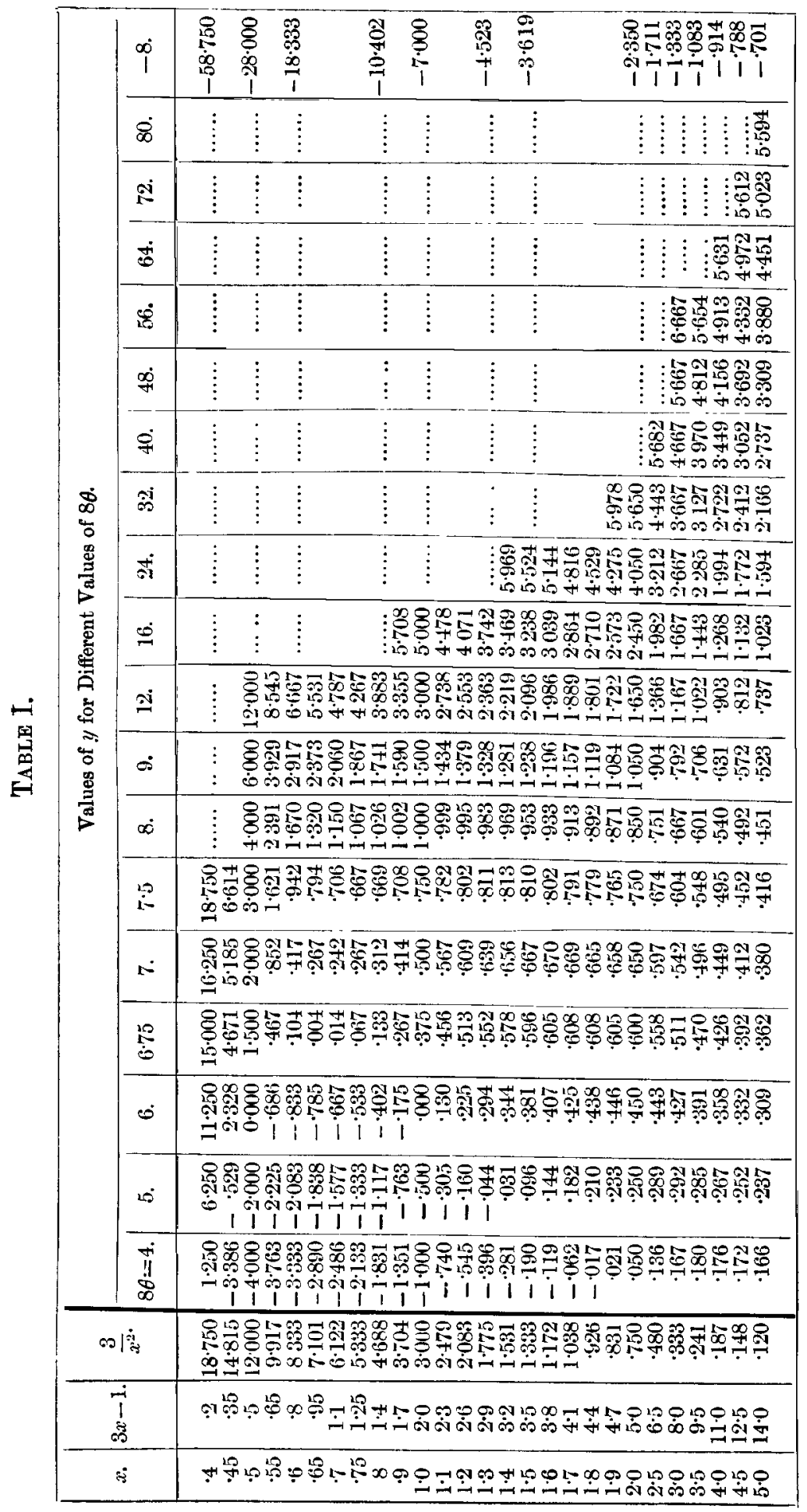


TABLE II.

\begin{tabular}{|c|c|c|c|c|}
\hline \multirow{2}{*}{$x$} & \multirow{2}{*}{$3 x-1$} & \multirow{2}{*}{$\frac{3}{3}$} & \multicolumn{2}{|c|}{$\begin{array}{c}\text { Values of } y \text { for Different } \\
\text { Values of } 8 \theta \text {. }\end{array}$} \\
\hline & & & $8 \theta=4$ & $8 \theta=-8$. \\
\hline $\begin{aligned} & \cdot 3 \\
& \cdot 25 \\
& .2 \\
& .15 \\
& \cdot 1 \\
&-\cdot 5 \\
&-1 \cdot 0 \\
&-1 \cdot 5 \\
&-2 \cdot 0 \\
&-2 \cdot 5 \\
&-3 \cdot 0 \\
&-3 \cdot 5 \\
&-4 \cdot 0 \\
&-4 \cdot 5 \\
&-5 \cdot 0\end{aligned}$ & 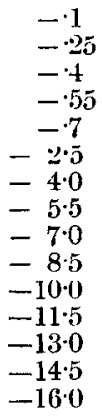 & $\begin{array}{r}33 \cdot 333 \\
48 \cdot 000 \\
75 \cdot 000 \\
133.333 \\
300 \cdot 000 \\
1 \cdot 000 \\
3 \cdot 000 \\
1 \cdot 333 \\
-750 \\
-480 \\
-333 \\
-241 \\
-187 \\
-148 \\
.120\end{array}$ & $\begin{array}{r}-73 \cdot 333 \\
-\quad 64 \cdot 000 \\
-85 \cdot 000 \\
-140606 \\
-305 \cdot 814 \\
-13 \cdot 600 \\
-4 \cdot 000 \\
-2 \cdot 060 \\
-1.321 \\
-.951 \\
-.733 \\
-.589 \\
-.495 \\
-.424 \\
-.370\end{array}$ & $\begin{array}{r}46.667 \\
-16 \cdot 000 \\
-55 \cdot 000 \\
-118 \cdot 788 \\
-288.571 \\
-8.800 \\
-1.000 \\
.121 \\
.393 \\
.461 \\
.467 \\
.455 \\
.428 \\
.404 \\
.380\end{array}$ \\
\hline
\end{tabular}

Differentiating $(\alpha)$ we have

$$
\frac{d y}{d x^{2}}=\frac{-24 \theta}{(3 x-1)^{2}}+\frac{6}{x^{3}}
$$

$\therefore$ The tangent is parallel to $y=0$ when $(3 x-1)^{2}=4 x^{3} \theta$. Eliminating $\theta$ between this equation and $(\alpha)$, we have

$$
(3 x-2)=y x^{3}, \quad \text {. . . . . . }(\beta)
$$

as the curve through the points where $y$ has a maximum or minimum value.

Differentiating $(\beta)$ we have

$$
\frac{d y}{d r^{r}}=\frac{\overrightarrow{b(1-x)}}{x^{4}} \text { and } \frac{d^{2} y}{d x^{2}}=\frac{6(3 x-4)}{x^{5}} ;
$$

$\therefore(\beta)$ has a tangent parallel to the axis of $x$ at point $(1,1)$ and an inflexion at the point $\left(\frac{4}{3}, \frac{2 \pi}{32}\right)$. This curve cuts $y=0$ where $x=\frac{2}{3}$, has a triple point at infinity at which tangents coincide with $x=0$, and has an inflexion at infinity at which the tangent is $y=0$. It is of degree 4 , class 4 , and deficiency zero; has 1 double point and 2 cusps (coinciding in the multiple point at infinity), 1 double tangent and 2 inflexions (one at infinity).

It passes through the points $(-6 ; 093)(-5 ; \cdot 136)$ $(-4 ; \cdot 169)(-3 ; \cdot 407)(-2 ; 1)(-1.5 ; 1 \cdot 926)(-1 ; 5)$ $(-.5 ; 28)(\cdot 1 ;-1700)(\cdot 2 ;-175)(\cdot 3 ;-40 \cdot 741)$ 
Mr. H. Hilton on van der Waals' Equation.

$(\cdot 333 ;-27)(\cdot 4 ;-12 \cdot 5)(\cdot 5 ;-4)(\cdot 667 ; 0)(\cdot 8 ; \cdot 781)$ $(1 ; 1)(1 \cdot 333 ; \cdot 844)(1 \cdot 5 ; \cdot 741) \quad(2 ; \cdot 5) \quad(2 \cdot 5 ; \cdot 352)$ $(3 ; 259)(4 ; \cdot 156)(5 ; \cdot 104)$, and is shown in fig. 1 . Differentiating $(\alpha)$ again twice we have

$$
\frac{d^{2} y}{d x^{2}}=\frac{144 \theta}{(3 x-1)^{3}}-\frac{18}{x^{4}} ; \quad \frac{d^{3} y}{d x^{3}}=\frac{-1296 \theta}{(3 x-1)^{4}}+\frac{72}{x^{5}} .
$$

$\therefore$ At a point of inflexion $(3 x-1)^{3}=8 \theta x^{4}$. Eliminating $\theta$ between this equation and ( $\alpha$ ) we have

$$
y x^{4}=6 x^{2}-6 x+1 \text {. . . . . }(\gamma)
$$

as the curve through the inflexions of the family. For a point where the tangent has 4 -point contact we must have

$$
\frac{d^{2} y}{d x^{2}}=\frac{d^{3} y}{d x^{3}}=0
$$

and hence we have $x=\frac{4}{3} ; \theta=\frac{2187}{2048}$; hence we see that the curve $(\gamma)$ touches the member of the firmily for which $\theta=\frac{2187}{2018}$ at the point $\left(\frac{4}{3} ; \frac{297}{256}\right)$; it also touches the branch $y x^{2}=-3$ of the curve of the family for which $\theta=0$, at the point $\left(\frac{1}{3} ;-27\right)$ (but elsewhere lies wholly above the branch), but touches no other member of the family at a finite point.

Differentiating $(\gamma)$ we have

$$
\frac{d y}{d x}=\frac{-2}{x^{2}}\left(6 x^{2}-9 x+2\right), \frac{d^{2} y}{d x^{2}}=\frac{4}{x^{6}}(3 x-5)(3 x-1) ;
$$

it has therefore tangents parallel to $y=0$ where $x=\cdot 271$ and 1.229 (approximately), and inflexions at the points $\left(\frac{1}{3} ;-27\right)$ $\left(\frac{5}{3} ; \cdot 9936\right)$.

It passes through the points $(-5 ; \cdot 290)(-4 ; \cdot 519)$ $(-3 ; \cdot 901)(-2 ; 2 \cdot 312)(-1 ; 13)(\cdot 1 ; 4600)(\cdot 2 ; 25)$ $(\cdot 211 ; 0)(\cdot 25 ;-68)(\cdot 3 ;-41 \cdot 975)(\cdot 333 ;-27)(\cdot 4 ;-17 \cdot 188)$ $(.5 ;-8)(\cdot 789 ; 0)(1 ; 1)(1 \cdot 2 ; 1 \cdot 178)(1 \cdot 333 ; 1 \cdot 160)$ $(1 \cdot 667 ; \cdot 994)(2 ; \cdot 812)(3 ; \cdot 457)(4 ; \cdot 285)(5 ; \cdot 194)$, and is shown (as far as possible) in fig. 1 .

It is of degree 5 , class 5 , and deficiency zero ; it has 3 double points and 3 cusps (coinciding in the multiple point at infinity) ; 3 bitangents (two of which are imaginary); and 3 inflexions (all real, one at infinity). It has a quadruple point at infinity at which the tangents coincide with $x=0$, and an inflexion at infinity at which the tangent is $y=0$.

It meets the curves of the family (a) for which $\theta=$ $\frac{9}{8}( \pm \sqrt{ } 3-1)[\cdot 824$ and -3074 approx. $]$ in the axis of $x$.

Phil. Mug. S. 6. Vol. 1. No. 5. May 1901. 2 Q 
The curves $(\beta)$ and $(\gamma)$ only meet in 2 finite points, namely $(1 ; 1)\left(\frac{1}{3} ;-27\right)$.

Their radii of curvature at the point whose abscissa is $x$ are respectively

$$
\frac{\left\{x^{8}+36(1-x)^{2}\right\}^{\frac{3}{2}}}{6 x^{7}(3 x-4)} \text { and } \frac{\left\{x^{10}+4\left(6 x^{2}-9 x+2\right)^{2}\right\}^{\frac{3}{2}}}{4 x^{9}(3 x-5)(3 x-1)} \text {. }
$$

If $\mathrm{ACE}$ is a straight line parallel to $y=0$, such that for a certain value of $\theta$ the areas $\mathrm{ABC}, \mathrm{EDC}$ are equal (see fig. 3); then the ordinate of $\mathrm{A}$ (or $\mathrm{E}$ ) represents the pressure

Fig. 3.

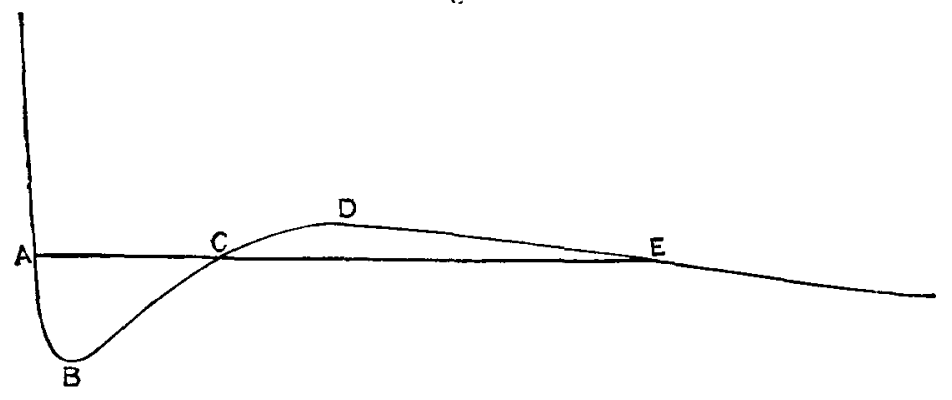

at which the substance boils for that particular value of $A$. The curve through all such points as $\mathrm{A}$ and $\mathrm{E}$ we will call the "border" curve. ( $C f$. Memoirs of Phys. Soc. London, vol. i. part 3, p. 453.) Let $x_{1}, x_{2}, x_{3}$ be the abscissæ of A, E, and $C$ respectively, and $y$ their ordinate. Then

$$
\left(y+\frac{3}{x_{2} x_{1}}\right)\left(x_{2}-x_{1}\right)=\frac{8 \theta}{3} \log \left(\frac{3 x_{2}-1}{3 x_{1}-1}\right) . \quad .
$$

[See Nernst's 'Theoretical Chemistry.']

Now $x_{1}, x_{2}, x_{3}$ are the roots of $3 y x^{3}-(y+8 \theta) x^{2}+9 x-3=0$, and therefore $\quad x_{1} x_{2}+x_{2} x_{3}+x_{5} x_{1}=\frac{3}{y} ; x_{1} x_{2} x_{3}=\frac{1}{y}$.

$\therefore$ eliminating $x_{3}, y x_{1}^{2} x_{2}^{2}+x_{1}+x_{2}-3 x_{1} x_{2}=0$, and therefore

$$
x_{2}=\frac{3 x_{1}-1+\sqrt{\left(3 x_{1}-1\right)^{2}-4 y x_{1}^{3}}}{2 y x_{1}^{2}}
$$

the positive sign being taken, for $y$ is positive in the region considered, and $x_{2}>x_{3}$.

Substituting this value for $x_{2}$ and $\frac{\left(y x_{1}^{2}+3\right)\left(3 x_{1}-1\right)}{x_{1}^{2}}$ 
$8 \theta$ in (i.) we have (writing $x$ for $x_{1}$ )

$$
\begin{gathered}
3\left(2 x^{2} y+9 x-3-3 \sqrt{1-6 x+9 x^{2}-4 y x^{3}}\right)\left(\sqrt{1-6 x+9 x^{2}-4 y x^{3}}-1+3 x-2 y x^{3}\right) \\
=4 y x^{2}\left(y x^{2}+3\right)(3 x-1) \times \log \left(\frac{3 \sqrt{1-6 x+9 x^{2}-4 y x^{3}}-3+9 x-2 y x^{2}}{2 y x^{2}(3 x-1)}\right)
\end{gathered}
$$

as the equation of the border curve between $x=\frac{1}{3}$ and $x=1$.

Similar reasoning shows that the equation of the border curve between $x=1$ and $x=\infty$ is found by changing the sign of the radical in $(\delta)$.

To trace the curve ( $\delta)$ directly is not easy, but we can readily determine a number of points on it with fair accuracy when once a number of curves of the family $(\alpha)$ are carefully drawn. We have in fact to choose the points A, E (fig. 3) so that the areas $\mathrm{ABC}, \mathrm{EDC}$ are equal, and this may be done by help of a planimeter ; or, more simply, by ensuring the equality of the number of millimetre squares in these two areas when the curves $(a)$ are plotted out on paper ruled in square millimetres.

The curve $(\delta)$ evidently passes through the points $(1 ; 1)$ and $\left(\frac{1}{3} ; 0\right)$ [as may be verified also from its equation]. The tangent at each point is parallel to the axis of $x:$ this is evident in the case of the former point, we may prove it in the case of the latter, thus :-A tangent to $(\alpha)$ parallel to the axis of $x$ is $y=\frac{8 \theta}{3 . t-1}-\frac{3}{x^{2}}$, where $(3 x-1)=4 \theta \cdot x^{3}$; if $\theta$ is small, $x$ must be large [supposing $3 x-1 \neq 0$ ], and we have approximately $4 \theta x=9$. Then the tangent becomes (substituting $\frac{9}{4 \theta}$ for $x$, and neglecting higher powers of $\theta$ than the second) $y=\frac{16}{27} \theta^{2}$. Hence the ordinate of the point on the border curve corresponding to this value of $\theta$ is $<\frac{16}{27} \theta^{2}$.

Now the curve $(\alpha)$ cuts $y=0$,

where

$$
x=\frac{9-9 \sqrt{1-\frac{32}{27} \theta}}{16 \theta}=\frac{1}{3}+\left(\frac{4}{27}\right)^{2} \theta,
$$

(if $\theta$ is small), and makes an angle

$$
\tan ^{-1}\left\{\frac{-24 \theta}{(3 x-1)^{2}}+\frac{6}{x^{3}}\right\} \text { or } \tan ^{-1}\left\{\frac{-3^{11}}{2^{5} \theta}+\frac{6}{x^{3}}\right\}
$$

or $\frac{\pi}{2}$ when $\theta$ is small, vith the axis of $x$; hence the abscissa 
588 Mr. H. Hilton on van der Waals' Equation.

of the point on $(\delta)$ corresponding to this value of $\theta$ is

$$
\frac{1}{3}+\left(\frac{4}{27}\right)^{2} \theta
$$

hence the tangent to $(\delta)$ at point $\left(\frac{1}{3} ; 0\right)$ makes an angle

$$
<\tan ^{-1}\left[\frac{\frac{16}{27} \theta^{2}}{\left(\frac{4}{27}\right)^{2} \theta}\right]_{\theta=0}
$$

with the axis of $x$; i.e., it touches the axis of $x$ at this point.

The curve $(\delta)$ is shown in fig. 1 .

The relation between the reduced temperature and volume at the boiling-point is found by eliminating $y$ between $(\alpha)$ and $(\delta)$; it is :-

$$
\begin{aligned}
& 3\left\{(16 \theta+27) x^{2}-36 x+9\right. \\
& \left.-3(3 x-1) \sqrt{\frac{(27-32 \theta) x^{3}+9 x^{2}-3 x-1}{3 x-1}}\right\}\left\{-16 x^{3} \theta+27 x^{2}-12 x+1\right.
\end{aligned}
$$$$
\left.+(3 x-1) \sqrt{\frac{(27-32 \theta) x^{3}+9 x^{2}-3 x-1}{3 x-1}}\right\}=32 x^{2}(3 x-1)\left(8 x^{2} \theta-9 x+3\right) \theta
$$$$
\times \log \left\{\frac{(27-16 \theta) x^{2}-3+3(3 x-1) \sqrt{\frac{(2 \pi-32 \theta) x^{3}+9 x^{2}-3 x-1}{3 x-1}}}{(3 x-1)\left(16 \theta x^{2}-18 x+6\right)}\right\}
$$

between $x=\frac{1}{3}$ and $x=1$; and the same with the sign of the radical changed between $x=1$ and $x=\infty$.

When $y$ is so large that the equation

$$
3 y x^{3}-(y+8 \theta) \cdot x^{2}+9 x-3=0
$$

has only one real root, this root is ( $f$. Works on Theory of Equations)

$$
\begin{aligned}
& \frac{1}{3 y}\left[\frac{y+8 \theta}{3}+\sqrt[3]{\frac{1}{2}\left\{18 y(y-4 \theta)+\frac{2}{27}(y+8 \theta)^{3}\right.}\right. \\
& \left.+2 y \sqrt{729 y+54\left(y^{2}-20 y \theta-8 \theta^{2}\right)+(y+8 \theta)^{3}}\right\}+\sqrt[3]{\frac{1}{2}\{18 y(y-4 y)} \\
& \left.\left.+\frac{2}{27}(y+8 \theta)^{3}-2 y \sqrt{729 y+54\left(y^{2}-20 y \theta-8 \theta^{2}\right)+(y+8 \theta)^{3}}\right\}\right]
\end{aligned}
$$

If we assume that this expression, which is the real root of $a$ when $y$ is large, by the principle of continuity represents the smallest root of the equation when $y$ becomes so small that the equation has three real roots, we obtain the relation be- 
tween the reduced pressure and temperature by substituting this expression for $x$ in the equation ( $\delta$ ).

It should be noted that we have confined ourselves to a strictly mathematical treatment without taking physical considerations into account. The equation $(\alpha)$ can hardly be considered to have any physical application when $\theta$ is negative, and ceases to represent necessarily the relation between the volume, pressure, and temperature of a substance when $x<\frac{2}{3}$, for then doubly central impacts between the molecules become impossible ( $f$. Memoirs of Phys. Soc. of London, rol. i. part 3, page 384 ).

References in the Figures.

\begin{tabular}{|c|c|c|c|c|c|c|c|c|c|c|c|c|c|c|c|c|c|c|c|c|}
\hline $\begin{array}{l}\text { Reterence } \\
\text { number... }\end{array}$ & 1 & 2 & 3 & 4 & 5 & 6 & 7 & 8 & 9 & 10 & 11 & 12 & 13 & 14 & 15 & 16 & 17 & 18 & 19 & 20 \\
\hline & 0 & 4 & 5 & 6 & 675 & 7 & $7 \cdot 5$ & 8 & 9 & 12 & 16 & 24 & 32 & 40 & 48 & 56 & 64 & 72 & 80 & -8 \\
\hline
\end{tabular}

All the curres in fig. 2 (except $\delta$ ) should run up to the asymptote $x=\frac{1}{3}$; but, for the sake of clearness, they are discontinued before they approach each other so closely as to be mutually indistinguishable.

Figs. 1 and 2 are ruled into reference squares whose sides are balf the unit of length.

Note. Since I wrote the above I hare heard from Mr. R. E. Baynes, M.A., Christchurch, Oxford, that he obtained an identical result for the equation of the border-curve many years ago.

LVII. On the Propagation of Cusped Wares and their Relation to ths Primary and Secondary Focal Lines. By Prof. R. W. WOOD*.

TN a previous paper (Phil. Mag. July 1900, p. 148) I have shown the forms of the wave-fronts reflected from spherical surfaces, by means of geometrical constructions, and photographs of the actual waves. In the present paper I shall discuss somewhat more fully the case of the reflexion of a plane wave by a hemispherical mirror, where we have a reflected wave of a form which $I$ bave likened to a volcanic cone. A superficial examination of the forms might lead one to imagine that the bowl of the crater collapsed to a point at the principal focus of the mirror. This can of course only be

* Communicated by the Physical Society. 